\title{
Numerical and asymptotic solutions of generalised Burgers' equation
}

\author{
Paul Hammerton and John Schofield
}

University of East Anglia

\begin{abstract}
The generalised Burgers' equation has been subject to a considerable amount of research on how the equation should behave according to asymptotic analysis, however there has been limited research verifying the asymptotic analysis. In order to verify the asymptotic analysis, this paper aims to run long time and detailed numerical simulations of Burgers' equation by employing suitable rescalings of Burgers' equation. It is hoped that this technique will make it possible to notice subtle changes in the shock structure which would otherwise be impossible to observe. The main aim of this paper is to validate the numerical methods used in order to allow further research into shock evolution where further relaxation effects will be included.
\end{abstract}

Keywords: Nonlinear Acoustics

PACS: $43.25 . \mathrm{Ba}$

\section{BACKGROUND AND BREAKDOWN OF WEAK SHOCK THEORY}

The generalised Burgers' equation is best known in the following form:

$$
\frac{\partial u}{\partial t}+u \frac{\partial u}{\partial x}=\varepsilon g(t) \frac{\partial^{2} u}{\partial x^{2}}
$$

where $\varepsilon \ll 1$ is the inverse Reynolds' number and $g(t)=1, \frac{t+t_{0}-1}{2}, \exp \left(\frac{t}{t_{0}}\right)$ is the term due to planar, cylindrical and spherical spreading respectively with $t_{0}$ a parameter related to the source amplitude. In this paper the aim is to investigate the leading order asymptotic behaviour and old age solutions. Crighton and Scott [1] considered asymptotic solutions of (1) in the small $\varepsilon$ limit for an initial $\mathrm{N}$-wave For moderate times the solution consists of a shock moving outwards with speed $t^{\frac{1}{2}}$ and the height decreasing as $t^{-\frac{1}{2}}$. To capture the internal structure of a moving shock requires sophisticated numerical techniques. Instead we use rescalings to ensure that we deal with a shock which is centred at \pm 1 with amplitude 1 by letting $X=t^{-\frac{1}{2}} x, U=t^{\frac{1}{2}} u$ and $T=\ln t$. This reduces (1) to:

$$
U_{T}=\left[\frac{U}{2}+\left(\frac{X}{2}-U\right) U_{X}+\varepsilon G(T) U_{X X}\right], \quad U(X, 0)= \begin{cases}X & \text { for }|X| \leq 1 \\ 0 & \text { for }|X|>1\end{cases}
$$

where $G(T)=1, \frac{\exp (T)}{2}, \exp (\exp (T))$ corresponding to the planar, cylindrical and spherical cases. The solution consists of an outer solution $U=X$ for $|X|<1$ together with Taylor [2] shocks at $X= \pm 1$. The leading order solution about $X=1$ is given by:

$$
U_{0}=\frac{1}{2}[1-\tanh (\theta)], \quad \theta=\frac{X-\left(1-\varepsilon \int g(T)\right)}{4 \varepsilon g(T)} .
$$


The agrees with the equation derived by Crighton and Scott [1] in equation (3.8). Weak shock theory can break down in three possible ways: The shock moves too far away from its original location (i.e. $\varepsilon \int g(T) d T=O(1)$ ), the shock width is no longer small compared to the size of the $\mathrm{N}$-wave (i.e. $\varepsilon g(T)=O(1)$ ), the leading order asymptotic solution is no longer significantly larger than the next order correction term.

In order to see when the third condition breaks down we need to obtain the next order behaviour of the modified Burgers' equation. We accomplish this by searching for a solution of the form $U=U_{0}+\varepsilon U_{1}$. $U_{1}$ is determined as a function involving $\theta$ and $G(T)$. The details of the solution are not included here for reasons of space but the result obtained agrees with the form obtained by Crighton and Scott [1] in equation (3.13). It is found that the third condition will break down when any one of; $\varepsilon \int g(T) d T, \varepsilon g(T)$, $\varepsilon g^{\prime}(T)=O(1)$. In due course we consider the hierarchy of breakdown for the cases of planar, cylindrical and spherical spreading and compare the results with the numerical solutions, but first we consider the old-age behaviour.

\section{OLD AGE BEHAVIOUR}

Returning to (1), as $t \rightarrow 0, u \rightarrow 0$ due to the dissipative term and as such we can ignore the nonlinear effects in (1). We now set $y=x\left(\varepsilon \int g\right)^{-\frac{1}{2}}$ (and $\left.\tau=t\right)$ to give:

$$
\frac{\int g d \tau}{g} u_{\tau}=\frac{y}{2} u_{y}+u_{y y}
$$

Now we have the equation in a form which is separable. Then writing $u(x, t)=Y(y) T(\tau)$ in (4) we arrive at the following equations:

$$
T^{\prime}=\frac{A g}{\int g} T, \quad Y^{\prime \prime}+\frac{y}{2} Y^{\prime}-A Y=0,
$$

where $A$ is a separation constant. The solution to (5) is:

$$
T(\tau)=B\left(\int g\right)^{A}, \quad Y=\exp \left(-\eta^{2}\right) f(\eta),
$$

where $\eta=\frac{y}{2}$ and $f$ satisfies the Hermite equation, $f^{\prime \prime}-2 \eta f+\lambda f=0$, where $\lambda=$ $-2(2 A+1)$. This standard equation has polynomial solutions (Hermite Polynomials) when $\lambda=2 n$ ( $\mathrm{n}$ an non-negative integer) and solutions that grow exponentially otherwise. Exponentially growing solutions are not physically appropriate, and hence we are restricted to $\lambda=0,2,4,6, \ldots$ which corresponds to $A=-0.5,-1,-1.5, \ldots$ The condition $Y(0)=0$ restricts us to the integer values of $A$, of which $A=-1$ is the slowest decaying and will dominate the behaviour at long times. We obtain that $A=-1, f(\eta)=\eta$ and:

$$
u(x, t)=D\left(x \exp \left(-\frac{x^{2}}{4 \varepsilon \int g d t}\right)\right)\left(\int g d t\right)^{-\frac{3}{2}},
$$

where $D=D(\varepsilon)$ is an undetermined constant dependent on the form of $g(t)$. Now we wish to numerically determine the constant for all three cases and compare the results to the asymptotic expressions found by Crighton and Scott [1] and Enflo [3]. 


\section{NUMERICAL SOLUTIONS}

The numerical method used here is a finite difference approach using an uneven mesh in order to provide higher resolution for the shock region solved by the Runge-Kutta method in order to advance forward in time. In this section we'll look at the three different types of breakdown of the weak-shock theory mentioned earlier. The asymptotic predictions were confirmed by numerical results. Here, we only present key results which best illustrate comparisons for the initial shock structure and old age solution.

From the leading order behaviour of (3) for the planar case we would expect the shock location to move along the path $1-\varepsilon T+O\left(\varepsilon^{2}\right)$ and this to breakdown when $T \approx O\left(\varepsilon^{-1}\right)$. The comparison of numerical and asymptotic results of the shock location is shown in Figure 1. By looking at the modified Burgers' equation we would expect the
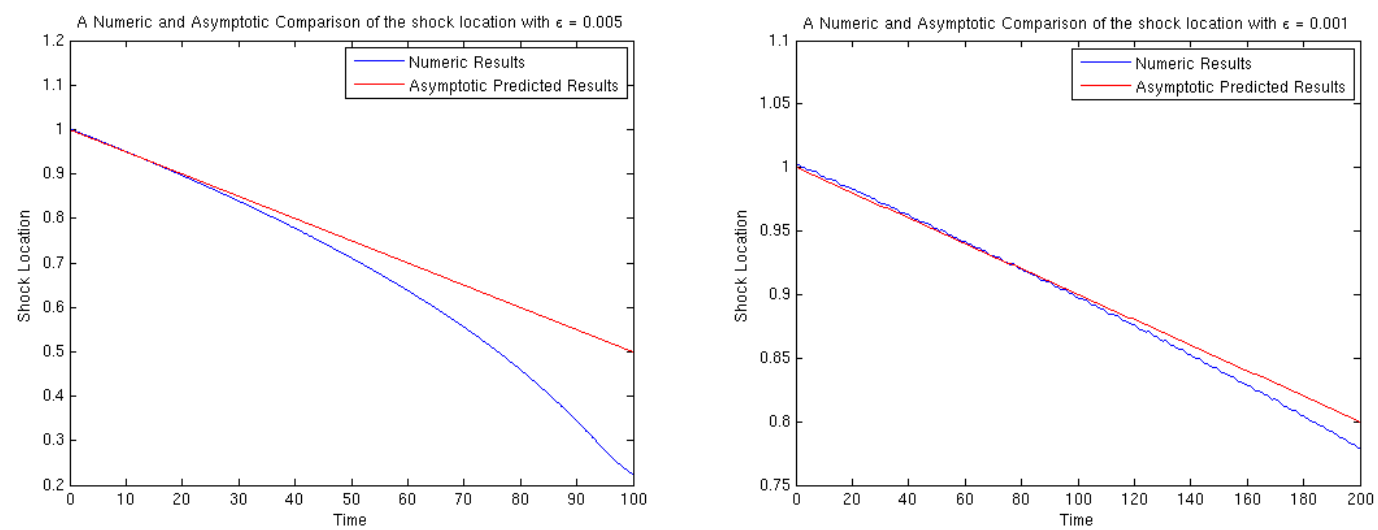

FIGURE 1. Asymptotic and Numerical Comparison of the shock location for the planar case with $\varepsilon=0.005$ and $\varepsilon=0.001$ with predicted breakdowns when $T \approx 200$ and 1000 respectively.

shock width to grow like $2 \varepsilon \exp (T)$ for the cylindrical case and the shock width to grow like $4 \varepsilon \exp (\exp (T))$ for the spherical case. The comparison of numerical and asymptotic behaviour for the cylindrical and spherical cases are shown in Figure 2. For the old age
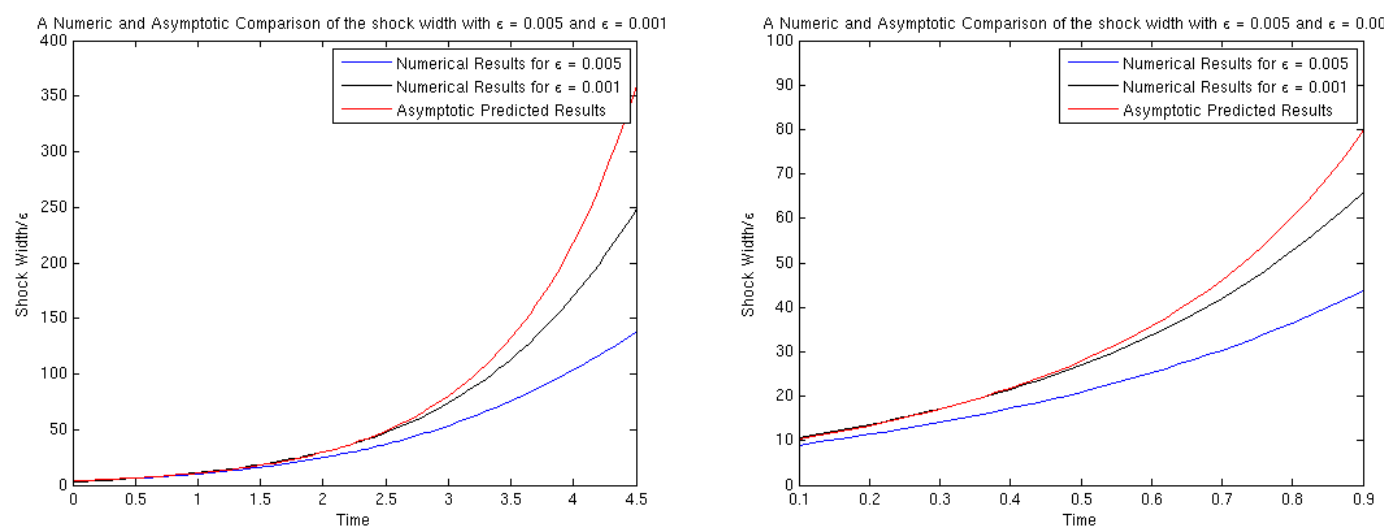

FIGURE 2. Asymptotic and Numerical Comparison for the normalised shock width for the cylindrical and spherical cases

solution in the cylindrical case, the constant $D$ has been determined by Enflo [3] through 

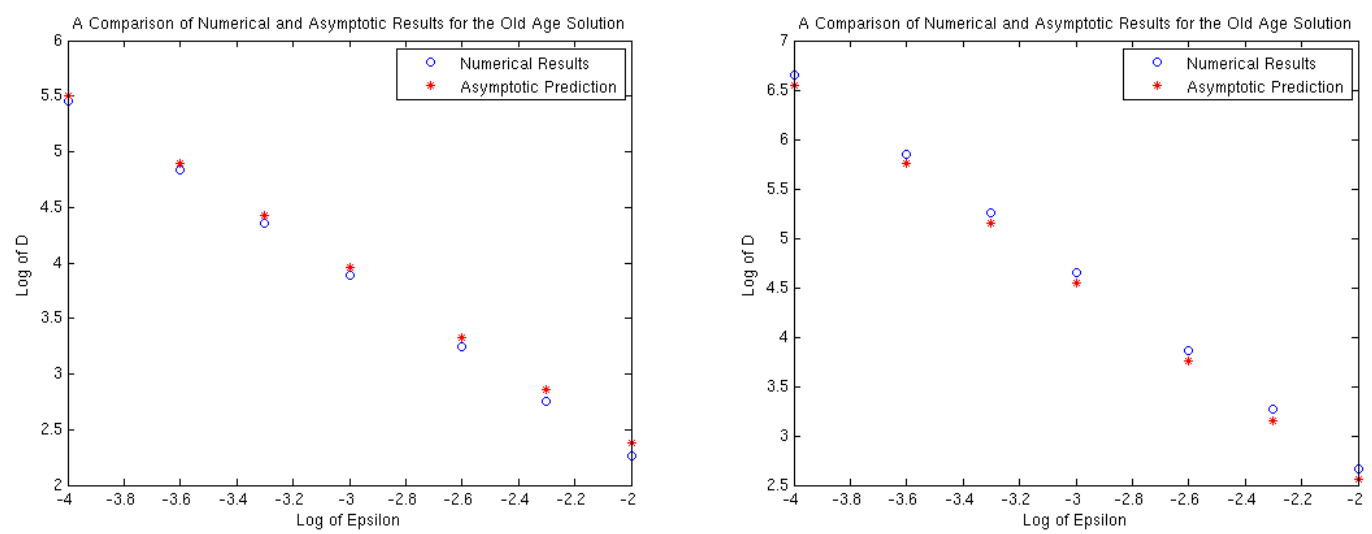

FIGURE 3. The values of $D$ for various values of $\varepsilon$ for the cylindrical and spherical cases.

matching the tail region through to the main shock region and is given by:

$$
D=\frac{1}{\varepsilon^{2}} \frac{27}{16 \sqrt{6 \mathrm{e}}}\left[1+2 \varepsilon-\frac{1}{2}\left(1+\frac{8}{3} \varepsilon\right)\left(\frac{\pi^{2}}{6}-\ln 2-\frac{11}{36}\right)\right] .
$$

For the spherical case, the constant $D$ has been found by Crighton and Scott [1] to be $C^{\frac{1}{2}}\left(6 \pi^{\frac{1}{2}} \varepsilon^{\frac{3}{2}}\right)^{-1}$ where $C$ is such that $\varepsilon \exp (C) C^{-1}=1$. Now the comparison between the asymptotic and numerical results for various values of $\varepsilon$ are shown in Figure 3.

\section{CONCLUSIONS}

In this paper extensive research has been undertaken to verify asymptotic results of Burgers' equations using a variable mesh. Particular time has been spent trying to verify the asymptotic predictions of the shock width and location using a numerical finite difference scheme. Also, long age solutions have been investigated and the asymptotic predictions have been found to hold true.

\section{REFERENCES}

1. D. G. Crighton, and J. F. Scott, Royal Society of London Philosophical Transactions Series A 292, 101-134 (1979).

2. G. I. Taylor, Royal Society of London Proceedings Series A 84, 371-377 (1910).

3. B. Enflo, Acta Acustica united with Acustica 84, 401-413 (1998). 